\section{Discussion}

This demographically atypical presentation of haemorrhage from gastric ulceration shows consistencies with observations previously described in the US. To our knowledge this is the first report in the UK of gastric ulceration complicating inhaled crack cocaine; a plausible mechanism underlying the pathogenesis being predictable from this illicit drug's pharmacology.

Gastric mucosal blood flow provides an important path for the disposal of influxing luminal acid, and thus an important barrier mechanism. ${ }^{4}$ Owing to cocaine's pharmacodynamics, namely monoaminergic synaptic potentiation (by inhibition of the uptake 1 transporter), and consequent alpha-1 adrenoceptor mediated mucosal arteriolar vasoconstriction, this presumably disrupts mucosal 'proton washout', predisposing to gastric ulceration. Recurrent short ischaemic episodes associated with frequent crack smoking would provide a background for prolonged impaired mucosal defence. The slow

1 Abramson DJ, Gertler JP, Lewis T, Kral JG. Crack related perforated gastropyloric ulcer. F Clin Gastroenterol 1991; 13: 17-9.

2 Lee HS, Lamaute HR, Pizzi WF, Prichard DL, Luks F. Acute gastrointestinal perforations associated with the use of crack. Ann Surg 1990; 211: 15-7.

3 Benowitz NL. Clinical pharmacology and toxicology of cocaine. Pharmacol Toxicol 1984; 72: 3-12
Summary points

- the pharmacokinetics of crack cocaine underly its specific complications

- gastrointestinal haemorrhage secondary to gastric peptic ulceration is a rare, potential complication of cocaine misuse

- peptic ulceration probably results from disruption of the gastric mucosal barrier mechanism through its sympathomimetic activity

absorption/low peak plasma kinetics of snorted cocaine hydrochloride probably explains the absence of such systemic toxic manifestations.

In view of the increasing use of this agent in Europe, ${ }^{5}$ similar presentations are likely to become more common. One scenario for example, being the recent upsurge in cocaine trafficking into the UK by 'body packers' (swallowers'), ${ }^{6}$ whereby package rupture or leak may potentially expose these individuals to the risk of such life-threatening complications.

4 Marrone GC, Silen W. Pathogenesis, diagnosis and treatment of gastric mucosal lesions. Clin Gastroenterol 1984; 13 635-51.

5 Strang J, Johns A, Caan W. Cocaine in the UK. $B r \mathcal{F}$ Psychiatry 1993, 162: 1-13.

6 Anon. Surgical aspects of international drug smuggling. (Editorial) BMY 1988; 296: 1035-7.

\title{
Spinal cord compression by spontaneous spinal subdural haematoma in polycythemia vera
}

\author{
Peter Kalina, Karl E Drehobl, Karen Black, Rona Woldenberg, Melissa Sapan
}

\begin{abstract}
Summary
A woman with an eight-year history of polycythemia vera presented with numbness and weakness of both legs. A large spinal haematoma was revealed on magnetic resonance imaging which was treated clinically and which subsequently resolved.
\end{abstract}

Keywords: polycythemia vera, spinal haematoma

Spontaneous, nontraumatic spinal epidural or subdural haematomas are uncommon and are a rare cause of spinal cord compression. Approximately half of spinal haematomas are thought to be spontaneous. Spontaneous bleeding may occur in polycythemia vera. However, polycythemia vera has not been previously described as a cause of spontaneous spinal haematoma.

\section{Case report}

A 60-year-old woman with an eight-year history of polycythemia vera presented with numbness and weakness of both lower extremities lasting 30 minutes. Although she had been maintained on phlebotomy on a regular basis, her platelet count was $1.25 \times 10^{6} / 1$. Emergency magnetic resonance imaging (MRI; figure) of the spine demonstrated a large subdural haematoma from T7-S2 with spinal cord compression. Due to the patient's bleeding tendency, it was elected to follow her clinically rather than perform surgical decompression. Treatment also included hydroxyurea, interferon and platelet pheresis. Her motor strength continued to improve and she was discharged. Follow-up outpatient MRI demonstrated resolution of the spinal haematoma. 


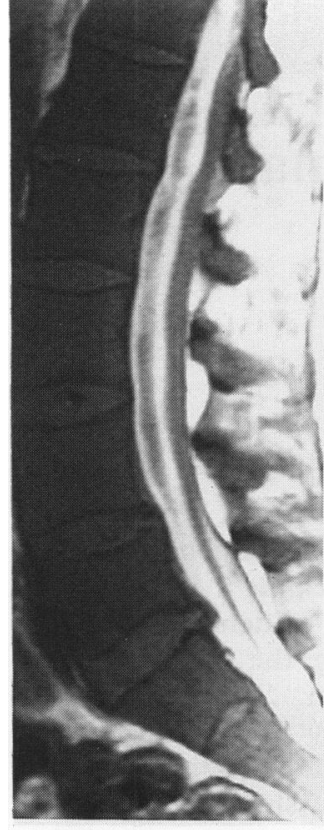

A

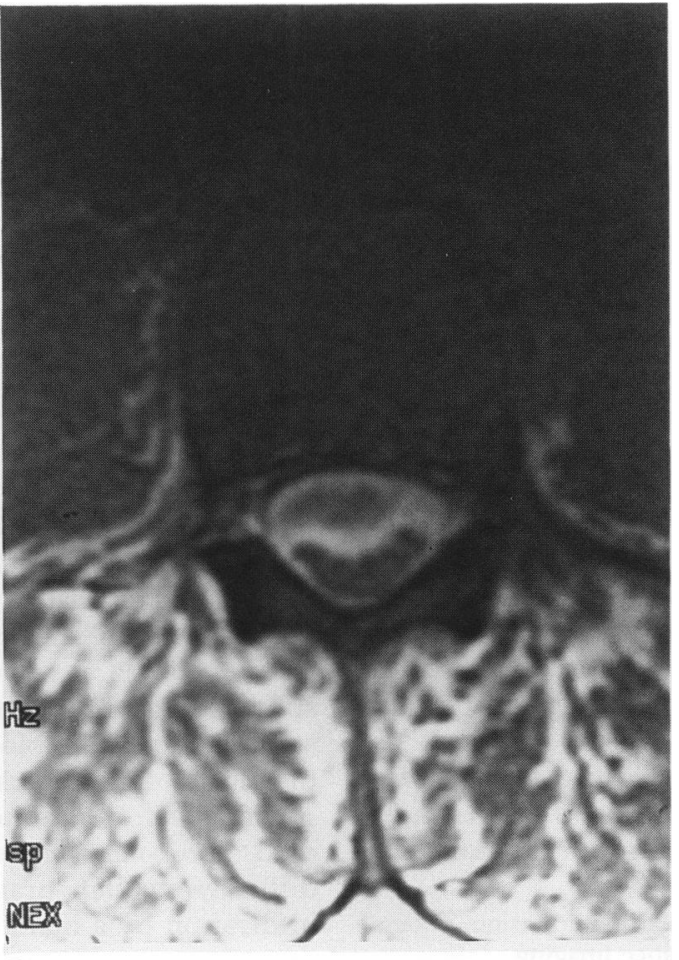

B

Figure (A) Sagittal $T_{1}$-weighted image 600/20/2 (TR/TE/excitations) shows an extensive hyperintense subacute haematoma within the ventral canal from $T 12$ to $S 2$, significantly compressing the thecal sac. Note the abnormal decreased signal of all vertebral bodies consistent with marrow hyperplasia. (B) Axial $T_{1}$-weighted image at L1 demonstrates the subdural haematoma compressing the conus

\section{Discussion}

Polycythemia vera is a myeloproliferative disorder manifested by overproduction of erythrocytes, granulocytes and megakaryocytes. The disease begins in middle life with a gradual onset and slowly progressive course. Patients present with symptoms secondary to expanded blood volume or with thrombotic or haemostatic complications. Splenomegaly is seen in $75 \%$ of cases. Laboratory analysis

\begin{tabular}{|l|}
\hline Polycythemia vera: features \\
\hline - myeloproliferative disorder \\
- splenomegaly \\
- elevated haemoglobin \\
- thated haematocrit \\
- leukocytocytosis \\
- spontaneous bleeding \\
\hline
\end{tabular}

1 Golde DW, Gulati SC. The myeloproliferative diseases. In: Isselbacher KJ, et al, eds. Harrison's principles of internal medicine, 13th edn. New York: McGraw-Hill, 1994; pp 1757-62.

2 Mittel RR, Meisler AI. Spontaneous massive abdominal wall hematoma in polycythemia vera. Acta Haematol 1984 72: $128-30$

3 Kheterpal S, Sayegh F, Cader A. A case of polycythemia vera presenting with intramuscular chest wall haematoma. Postgrad Med $\mathcal{F}$ 1992; 68: 381 -2.

4 Mackenzie JW, Jellicoe JA. Acute upper airway obstruction spontaneous retropharyngeal haematoma in a patient with polycythemia rubra vera. Anaesthesia 1986; 41: 57-9. reveals increased haemoglobin, increased haematocrit, thrombocytosis and leukocytosis. Phlebotomy is used to lower the haematocrit to under $46 \%$ so that survival greater than 12 years is now common. ${ }^{1}$

Polycythemia vera may present with its most common complications of thrombosis or haemorrhage. Bleeding, which is less common than thrombosis, usually occurs in the gastrointestinal tract, epistaxis or ecchymosis. ${ }^{2,4}$ There have been several reports of spontaneous bleeding in polycythemia vera including retropharyngeal, mediastinal, abdominal wall, intrahepatic and chest wall. ${ }^{2-5}$ The haemorrhagic tendency in polycythemia vera may be related to decreased platelet adhesion, defective platelet aggregation, chronic disseminated intravascular coagulation with excess production of hyperaggregated platelets or the release of ineffective platelets. ${ }^{3}$ Nonspontaneous aetiologies of spinal haematoma include trauma, coagulopathy, neoplasm, vascular malformation or iatrogenic. ${ }^{6}$ Other theories for spontaneous spinal haematoma include increased pressure in the epidural venous or arterial plexus resulting in rupture and haemorrhage. ${ }^{6} \mathrm{MRI}$ may be used to differentiate the similar appearances of subdural vs epidural haematoma. While the inverted 'Mercedes sign' has been reported as suggestive of subdural haematoma, ${ }^{7}$ this finding is not classically demonstrated in our case.

Spontaneous bleeding in unusual sites may be secondary to underlying polycythemia vera. Spinal haematoma should be suspected in patients complaining of back and radicular pain with motor and sensory deficits related to a specific level of compression. Prompt surgical evacuation may result in resolution of the neurologic deficits. However, the increased bleeding tendency in polycythemia vera may warrant following the patient clinically and radiographically without surgery.

\section{Learning/summary points}

spontaneous spinal haematoma is a rare cause of spinal cord compression

- half of spinal haematomas are spontaneous

- spinal haematoma should be suspected in patients complaining of back or radicular pain or motor and sensory deficits

- MRI may be used to differentiate subdural from epidural haematoma

- spontaneous bleeding in unusual sites may be secondary to underlying polycythemia vera

- polycythemia vera may be a cause of spontaneous spinal haematoma

5 Isles C, Strachan RW. Polycythemia vera presenting as an acute abdomen. Postgrad Med F 1981; 57: 314-6.

6 Joseph AP, Vinen JD. Acute spinal epidural haematoma. $f$ Emerg Med 1993; 11: 437-41.

7 Johnson PJ, Hahn F, McConnell J, Graham EG, Leibrock LG. The importance of MRI findings for the diagnosis of nontraumatic lumbar subacute subdural haematomas. Acta Neurochirurg 1991; 113: 186-8. 